Colloq. Math. 130(2013), no. 1, 67-78.

\title{
ARITHMETIC THEORY OF HARMONIC NUMBERS (II)
}

\author{
ZHI-Wei SuN ${ }^{1}$ AND Li-Lu ZhaO ${ }^{2}$ \\ ${ }^{1}$ Department of Mathematics, Nanjing University \\ Nanjing 210093, People's Republic of China \\ zwsun@nju.edu.cn \\ http://math.nju.edu.cn/ zwsun
}

${ }^{2}$ School of Mathematics

Hefei University of Technology

Hefei 230009, People's Republic of China

zhaolilu@gmail.com

\begin{abstract}
For $k=1,2, \ldots$ let $H_{k}$ denote the harmonic number $\sum_{j=1}^{k} 1 / j$. In this paper we establish some new congruences involving harmonic numbers. For example, we show that for any prime $p>3$ we have

$$
\sum_{k=1}^{p-1} \frac{H_{k}}{k 2^{k}} \equiv \frac{7}{24} p B_{p-3} \quad\left(\bmod p^{2}\right), \quad \sum_{k=1}^{p-1} \frac{H_{k, 2}}{k 2^{k}} \equiv-\frac{3}{8} B_{p-3} \quad(\bmod p),
$$

and

$$
\sum_{k=1}^{p-1} \frac{H_{k, 2 n}^{2}}{k^{2 n}} \equiv \frac{\left(\begin{array}{l}
6 n+1 \\
2 n-1
\end{array}\right)+n}{6 n+1} p B_{p-1-6 n}\left(\bmod p^{2}\right)
$$

for any positive integer $n<(p-1) / 6$, where $B_{0}, B_{1}, B_{2}, \ldots$ are Bernoulli numbers, and $H_{k, m}:=\sum_{j=1}^{k} 1 / j^{m}$.
\end{abstract}

\section{INTRODUCTION}

Recall that harmonic numbers are those

$$
H_{n}:=\sum_{0<k \leqslant n} \frac{1}{k} \quad(n \in \mathbb{N}=\{0,1,2, \ldots\}),
$$

where $H_{0}:=0$ since we consider the value of an empty sum as zero. They play important roles in mathematics. In $1862 \mathrm{~J}$. Wolstenholme [W] showed

2010 Mathematics Subject Classification. Primary 11A07, 11B68; Secondary 05A19, $11 \mathrm{~B} 75$.

Keywords. Harmonic numbers, congruences, Bernoulli numbers.

The first author is supported by the National Natural Science Foundation (grant 11171140) of China and the PAPD of Jiangsu Higher Education Institutions. 
the congruence $H_{p-1} \equiv 0\left(\bmod p^{2}\right)$ for any prime $p>3$. Throughout this paper, for a prime $p$ and two rational $p$-adic integers $A$ and $B$, we write $A \equiv B\left(\bmod p^{n}\right)($ with $n \in \mathbb{N})$ to mean that $A-B$ is divisible by $p^{n}$ in the ring of $p$-adic integers.

In $[\mathrm{Su}]$ the first author investigated arithmetic properties of harmonic numbers systematically. For example, he proved that for any prime $p>5$ we have

$$
\sum_{k=1}^{p-1} \frac{H_{k}}{k 2^{k}} \equiv \sum_{k=1}^{p-1} \frac{H_{k}^{2}}{k^{2}} \equiv 0(\bmod p) .
$$

For $m \in \mathbb{Z}^{+}=\{1,2,3, \ldots\}$, harmonic numbers of order $m$ are defined by

$$
H_{n, m}:=\sum_{0<k \leqslant n} \frac{1}{k^{m}} \quad(n \in \mathbb{N}) .
$$

It is known that

$$
\sum_{k=1}^{\infty} \frac{H_{k}}{k 2^{k}}=\frac{\pi^{2}}{12} \quad \text { (S. W. Coffman [C], 1987) }
$$

and

$$
\sum_{k=1}^{\infty} \frac{H_{k, 2}}{k 2^{k}}=\frac{5}{8} \zeta(3) \quad \text { (B. Cloitre, 2004). }
$$

Both identities can be found in [SW].

Our first theorem is as follows.

Theorem 1.1. For any prime $p>3$, we have

$$
\sum_{k=1}^{p-1} \frac{H_{k}}{k 2^{k}} \equiv \frac{7}{24} p B_{p-3} \quad\left(\bmod p^{2}\right)
$$

and

$$
\sum_{k=1}^{p-1} \frac{H_{k, 2}}{k 2^{k}} \equiv-\frac{3}{8} B_{p-3}(\bmod p),
$$

where $B_{0}, B_{1}, B_{2}, \ldots$ are Bernoulli numbers.

Remark 1.1. (1.1) confirms the first part of [Su, Conjecture 1.1]. The second part of $\left[\mathrm{Su}\right.$, Conjecture 1.1] states that $\sum_{k=1}^{p-1} H_{k}^{2} / k^{2} \equiv \frac{4}{5} p B_{p-5}$ $\left(\bmod p^{2}\right)$ for any prime $p>3$; this was confirmed by R. Meštrović $[\mathrm{M}]$ quite recently.

Our second theorem confirms the second conjecture of $[\mathrm{Su}]$. 
Theorem 1.2 ([Su, Conjecture 1.2]). Let $p$ be an odd prime and let $n$ be a positive integer with $p-1 \nmid 6 n$. Then

$$
\sum_{k=1}^{p-1} \frac{H_{k, 2 n}^{2}}{k^{2 n}} \equiv 0(\bmod p)
$$

Furthermore, when $p>6 n+1$ we have

$$
\sum_{k=1}^{p-1} \frac{H_{k, 2 n}^{2}}{k^{2 n}} \equiv \frac{s(n)}{6 n+1} p B_{p-1-6 n}\left(\bmod p^{2}\right)
$$

where

$$
s(n)=\left(\begin{array}{c}
6 n+1 \\
2 n-1
\end{array}\right)+n
$$

Remark 1.2. We give here four initial values of the integer sequence $\{s(n)\}_{n \geqslant 1}$ :

$$
s(1)=8, s(2)=288, s(3)=11631, s(4)=480704 .
$$

We will show Theorems 1.1 and 1.2 in Sections 2 and 3 respectively.

\section{Proof of Theorem 1.1}

Lemma 2.1. Let $p>3$ be a prime. Then

$$
\sum_{k=1}^{p-1} \frac{(-1)^{k}}{k^{2}} \equiv \frac{p}{2} B_{p-3} \quad\left(\bmod p^{2}\right), \sum_{k=1}^{p-1} \frac{(-1)^{k}}{k^{3}} \equiv-\frac{B_{p-3}}{2} \quad(\bmod p)
$$

and

$$
\sum_{k=1}^{p-1} \frac{H_{k}}{k} \equiv \frac{p}{3} B_{p-3} \quad\left(\bmod p^{2}\right) \quad \text { and } \sum_{k=1}^{p-1} \frac{(-1)^{k}}{k^{2}} H_{k} \equiv-\frac{B_{p-3}}{4} \quad(\bmod p) .
$$

Proof. It is known that (cf. [S, Corollaries 5.1 and 5.2])

$$
\sum_{k=1}^{p-1} \frac{1}{k^{2}} \equiv \frac{2}{3} p B_{p-3} \quad\left(\bmod p^{2}\right), \quad \sum_{k=1}^{p-1} \frac{1}{k^{3}} \equiv \frac{3}{4} p B_{p-4} \equiv-p \delta_{p, 5} \quad\left(\bmod p^{2}\right),
$$

and

$$
\sum_{k=1}^{(p-1) / 2} \frac{1}{k^{2}} \equiv \frac{7}{3} p B_{p-3} \quad\left(\bmod p^{2}\right) \text { and } \quad \sum_{k=1}^{(p-1) / 2} \frac{1}{k^{3}} \equiv-2 B_{p-3} \quad(\bmod p) .
$$


Thus

$$
\begin{aligned}
\sum_{k=1}^{p-1} \frac{(-1)^{k}}{k^{2}} & =\sum_{k=1}^{p-1} \frac{1+(-1)^{k}}{k^{2}}-\sum_{k=1}^{p-1} \frac{1}{k^{2}}=\frac{1}{2} H_{(p-1) / 2,2}-H_{p-1,2} \\
& \equiv \frac{7}{6} p B_{p-3}-\frac{2}{3} p B_{p-3}=\frac{p}{2} B_{p-3}\left(\bmod p^{2}\right)
\end{aligned}
$$

and

$$
\begin{aligned}
\sum_{k=1}^{p-1} \frac{(-1)^{k}}{k^{3}} & =\sum_{k=1}^{p-1} \frac{1+(-1)^{k}}{k^{3}}-\sum_{k=1}^{p-1} \frac{1}{k^{3}} \\
& =\frac{1}{4} H_{(p-1) / 2,3}-H_{p-1,3} \equiv \frac{-2 B_{p-3}}{4}(\bmod p) .
\end{aligned}
$$

Therefore (2.1) holds.

By the proof of [S, Theorem 6.1],

$$
\sum_{1 \leqslant j<k \leqslant p-1} \frac{1}{j k} \equiv-\frac{p}{3} B_{p-3} \quad\left(\bmod p^{2}\right) .
$$

So we have

$\sum_{k=1}^{p-1} \frac{H_{k}}{k}=\sum_{k=1}^{p-1} \frac{1}{k^{2}}+\sum_{1 \leqslant j<k \leqslant p-1} \frac{1}{j k} \equiv \frac{2}{3} p B_{p-3}-\frac{p}{3} B_{p-3}=\frac{p}{3} B_{p-3} \quad\left(\bmod p^{2}\right)$.

This proves the first congruence in (2.2).

Now we prove the second congruence in (2.2). Since

$$
H_{p-k}=H_{p-1}-\sum_{j=1}^{k-1} \frac{1}{p-j} \equiv H_{k-1}=H_{k}-\frac{1}{k} \quad(\bmod p)
$$

for all $k=1, \ldots, p-1$, we have

$$
\sum_{k=1}^{p-1} \frac{(-1)^{k}}{k^{2}} H_{k}=\sum_{k=1}^{p-1} \frac{(-1)^{p-k}}{(p-k)^{2}} H_{p-k} \equiv-\sum_{k=1}^{p-1} \frac{(-1)^{k}}{k^{2}}\left(H_{k}-\frac{1}{k}\right) \quad(\bmod p)
$$

and hence

$$
\sum_{k=1}^{p-1} \frac{(-1)^{k}}{k^{2}} H_{k} \equiv \frac{1}{2} \sum_{k=1}^{p-1} \frac{(-1)^{k}}{k^{3}} \equiv-\frac{B_{p-3}}{4} \quad(\bmod p)
$$

The proof of Lemma 2.1 is now complete. 
Lemma 2.2. (i) For any positive integers $k$ and $m$ we have

$$
\sum_{n=1}^{m}\left(\begin{array}{l}
n-1 \\
k-1
\end{array}\right)=\left(\begin{array}{l}
m \\
k
\end{array}\right)
$$

(ii) For each $n=1,2,3, \ldots$ we have

$$
\sum_{k=1}^{n}\left(\begin{array}{l}
n \\
k
\end{array}\right) \frac{(-1)^{k-1}}{k} H_{k}=H_{n, 2}
$$

Proof. (2.3) is well known (cf. [G, (1.5)]) and it can be easily proved by induction on $m$.

(2.4) is also known (cf. [H]). Here we prove it by induction. Clearly (2.4) holds for $n=1$. Assume that (2.4) holds for a fixed positive integer $n$. Then

$$
\begin{aligned}
\sum_{k=1}^{n+1}\left(\begin{array}{c}
n+1 \\
k
\end{array}\right) \frac{(-1)^{k-1}}{k} H_{k} & =\sum_{k=1}^{n}\left(\begin{array}{l}
n \\
k
\end{array}\right) \frac{(-1)^{k-1}}{k} H_{k}+\sum_{k=1}^{n+1}\left(\begin{array}{c}
n \\
k-1
\end{array}\right) \frac{(-1)^{k-1}}{k} H_{k} \\
& =H_{n, 2}+\frac{1}{n+1} \sum_{k=0}^{n+1}\left(\begin{array}{c}
n+1 \\
k
\end{array}\right)(-1)^{k-1} H_{k} .
\end{aligned}
$$

Note that

$$
\begin{aligned}
& \sum_{k=0}^{n+1}\left(\begin{array}{c}
n+1 \\
k
\end{array}\right)(-1)^{k-1} H_{k} \\
= & \sum_{k=0}^{n}\left(\begin{array}{l}
n \\
k
\end{array}\right)(-1)^{k-1} H_{k}+\sum_{k=1}^{n+1}\left(\begin{array}{c}
n \\
k-1
\end{array}\right)(-1)^{k-1}\left(H_{k-1}+\frac{1}{k}\right) \\
= & \sum_{k=1}^{n+1}\left(\begin{array}{c}
n \\
k-1
\end{array}\right) \frac{(-1)^{k-1}}{k}=-\frac{1}{n+1} \sum_{k=1}^{n+1}\left(\begin{array}{c}
n+1 \\
k
\end{array}\right)(-1)^{k}=\frac{1}{n+1} .
\end{aligned}
$$

So

$$
\sum_{k=1}^{n+1}\left(\begin{array}{c}
n+1 \\
k
\end{array}\right) \frac{(-1)^{k-1}}{k} H_{k}=H_{n, 2}+\frac{1}{n+1} \cdot \frac{1}{n+1}=H_{n+1,2}
$$

as desired.

Lemma 2.3. Let $p>3$ be a prime. Then

$$
\sum_{1 \leqslant j \leqslant k \leqslant p-1} \frac{2^{j}(j+k)}{j^{2} k^{2}} \equiv \sum_{k=1}^{p-1} \frac{(-1)^{k}}{k^{3}}(\bmod p) .
$$


Proof. Observe that

$$
\begin{aligned}
& \sum_{1 \leqslant i \leqslant j \leqslant k \leqslant p-1} \frac{2^{i}}{i j k}-\sum_{1 \leqslant i<j<k \leqslant p-1} \frac{2^{i}}{i j k} \\
= & \sum_{1 \leqslant j \leqslant k \leqslant p-1} \frac{2^{j}}{j^{2} k}+\sum_{1 \leqslant i \leqslant j \leqslant p-1} \frac{2^{i}}{i j^{2}}-\sum_{k=1}^{p-1} \frac{2^{k}}{k^{3}} \\
= & \sum_{1 \leqslant j \leqslant k \leqslant p-1}\left(\frac{2^{j}}{j^{2} k}+\frac{2^{j}}{j k^{2}}\right)-\sum_{k=1}^{p-1} \frac{2^{k}}{k^{3}} .
\end{aligned}
$$

Similarly,

$$
\begin{aligned}
& 2 \sum_{1 \leqslant i \leqslant j \leqslant k \leqslant p-1} \frac{(-1)^{i}}{i j k}-2 \sum_{1 \leqslant i<j<k \leqslant p-1} \frac{(-1)^{i}}{i j k}-2 \sum_{k=1}^{p-1} \frac{(-1)^{k}}{k^{3}} \\
= & 2 \sum_{1 \leqslant j<k \leqslant p-1}\left(\frac{(-1)^{j}}{j^{2} k}+\frac{(-1)^{j}}{j k^{2}}\right) \\
\equiv & \sum_{1 \leqslant j<k \leqslant p-1}\left(\frac{(-1)^{j}}{j^{2} k}+\frac{(-1)^{j}}{j k^{2}}+\frac{(-1)^{p-j}}{(p-j)^{2}(p-k)}+\frac{(-1)^{p-j}}{(p-j)(p-k)^{2}}\right) \\
= & \sum_{1 \leqslant j<k \leqslant p-1} \frac{(-1)^{j}}{j^{2} k}+\sum_{1 \leqslant k<j \leqslant p-1} \frac{(-1)^{j}}{j^{2} k} \\
& +\sum_{1 \leqslant j<k \leqslant p-1} \frac{(-1)^{j}}{j k^{2}}+\sum_{1 \leqslant k<j \leqslant p-1} \frac{(-1)^{j}}{j k^{2}} \\
= & H_{p-1} \sum_{j=1}^{p-1} \frac{(-1)^{j}}{j^{2}}+H_{p-1,2} \sum_{j=1}^{p-1} \frac{(-1)^{j}}{j}-2 \sum_{j=1}^{p-1} \frac{(-1)^{j}}{j^{3}}(\bmod p) .
\end{aligned}
$$

Thus, with the help of $H_{p-1} \equiv H_{p-1,2} \equiv 0(\bmod p)$, we have

$$
\sum_{1 \leqslant i \leqslant j \leqslant k \leqslant p-1} \frac{(-1)^{i}}{i j k} \equiv \sum_{1 \leqslant i<j<k \leqslant p-1} \frac{(-1)^{i}}{i j k}(\bmod p) .
$$

By [ZS, Theorem 1.2],

$$
\sum_{1 \leqslant i<j<k \leqslant p-1} \frac{(1-x)^{i}}{i j k} \equiv \sum_{1 \leqslant i<j<k \leqslant p-1} \frac{x^{i}}{i j k} \quad(\bmod p) .
$$

So, in view of the above, we have

$$
\begin{aligned}
\sum_{1 \leqslant i \leqslant j \leqslant k \leqslant p-1} \frac{(-1)^{i}}{i j k} & \equiv \sum_{1 \leqslant i<j<k \leqslant p-1} \frac{2^{i}}{i j k} \\
& \equiv \sum_{1 \leqslant i \leqslant j \leqslant k \leqslant p-1} \frac{2^{i}}{i j k}+\sum_{k=1}^{p-1} \frac{2^{k}}{k^{3}}-\sum_{1 \leqslant j \leqslant k \leqslant p-1} \frac{2^{j}(j+k)}{j^{2} k^{2}}(\bmod p) .
\end{aligned}
$$


It remains to show that

$$
\sum_{1 \leqslant i \leqslant j \leqslant k \leqslant p-1} \frac{2^{i}-(-1)^{i}}{i j k} \equiv \sum_{k=1}^{p-1} \frac{(-1)^{k}-2^{k}}{k^{3}} \quad(\bmod p) .
$$

With the help of Lemma 2.2, we have

$$
\begin{aligned}
& \sum_{1 \leqslant i \leqslant j \leqslant k \leqslant p-1} \frac{2^{i}-(-1)^{i}}{i j k}=\sum_{1 \leqslant i \leqslant j \leqslant k \leqslant p-1} \frac{1}{i j k} \sum_{r=0}^{i}\left(1-(-2)^{r}\right)\left(\begin{array}{c}
i \\
r
\end{array}\right) \\
&=\sum_{r=1}^{p-1} \frac{1-(-2)^{r}}{r} \sum_{1 \leqslant j \leqslant k \leqslant p-1} \frac{1}{j k} \sum_{i=1}^{j}\left(\begin{array}{l}
i-1 \\
r-1
\end{array}\right) \\
&=\sum_{r=1}^{p-1} \frac{1-(-2)^{r}}{r} \sum_{1 \leqslant j \leqslant k \leqslant p-1} \frac{1}{j k}\left(\begin{array}{l}
j \\
r
\end{array}\right) \\
&=\sum_{r=1}^{p-1} \frac{1-(-2)^{r}}{r^{2}} \sum_{k=1}^{p-1} \frac{1}{k} \sum_{j=1}^{k}\left(\begin{array}{l}
j-1 \\
r-1
\end{array}\right) \\
&=\sum_{r=1}^{p-1} \frac{1-(-2)^{r}}{r^{2}} \sum_{k=1}^{p-1} \frac{1}{k}\left(\begin{array}{l}
k \\
r
\end{array}\right)=\sum_{r=1}^{p-1} \frac{1-(-2)^{r}}{r^{3}} \sum_{k=1}^{p-1}\left(\begin{array}{l}
k-1 \\
r-1
\end{array}\right) \\
&=\sum_{r=1}^{p-1} \frac{1-(-2)^{r}}{r^{3}}\left(\begin{array}{l}
p-1 \\
r
\end{array}\right) \equiv \sum_{r=1}^{p-1}(-1)^{r}-2^{r} \\
& r^{3} \\
&(\bmod p) .
\end{aligned}
$$

This proves the desired (2.6).

Proof of Theorem 1.1. We prove (1.2) first. In view of (2.4), we have

$$
\begin{aligned}
\sum_{n=1}^{p-1} \frac{H_{n, 2}}{n 2^{n}} & =\sum_{n=1}^{p-1} \frac{1}{n 2^{n}} \sum_{k=1}^{n}\left(\begin{array}{l}
n \\
k
\end{array}\right) \frac{(-1)^{k-1}}{k} H_{k} \\
& =\sum_{k=1}^{p-1} \frac{(-1)^{k-1}}{k} H_{k} \sum_{n=k}^{p-1} \frac{1}{n 2^{n}}\left(\begin{array}{l}
n \\
k
\end{array}\right)=\sum_{k=1}^{p-1} \frac{(-1)^{k-1}}{k^{2} 2^{k}} H_{k} \sum_{n=k}^{p-1}\left(\begin{array}{l}
n-1 \\
k-1
\end{array}\right) \frac{1}{2^{n-k}} \\
& =\sum_{k=1}^{p-1} \frac{(-1)^{k-1}}{k^{2} 2^{k}} H_{k} \sum_{j=0}^{p-1-k}\left(\begin{array}{c}
k+j-1 \\
j
\end{array}\right) \frac{1}{2^{j}} \\
& =\sum_{k=1}^{p-1} \frac{(-1)^{k-1}}{k^{2} 2^{k}} H_{k} \sum_{j=0}^{p-1-k}\left(\begin{array}{c}
-k \\
j
\end{array}\right) \frac{1}{(-2)^{j}}
\end{aligned}
$$


and hence

$$
\begin{aligned}
\sum_{n=1}^{p-1} \frac{H_{n, 2}}{n 2^{n}} & \equiv \sum_{k=1}^{p-1} \frac{(-1)^{k-1}}{k^{2} 2^{k}} H_{k} \sum_{j=0}^{p-1-k}\left(\begin{array}{c}
p-k \\
j
\end{array}\right) \frac{1}{(-2)^{j}} \\
& =\sum_{k=1}^{p-1} \frac{(-1)^{k-1}}{k^{2} 2^{k}} H_{k} \frac{1+(-1)^{k}}{2^{p-k}} \\
& \equiv-\frac{1}{2} \sum_{k=1}^{p-1} \frac{H_{k}}{k^{2}}\left(1+(-1)^{k}\right)(\bmod p) .
\end{aligned}
$$

Note that

$$
\sum_{k=1}^{p-1} \frac{H_{k}}{k^{2}} \equiv B_{p-3}(\bmod p) \text { and } \sum_{k=1}^{p-1} \frac{(-1)^{k}}{k^{2}} H_{k} \equiv-\frac{B_{p-3}}{4}(\bmod p)
$$

by $[\mathrm{ST},(5.4)]$ and $(2.2)$ respectively. So we get

$$
\sum_{n=1}^{p-1} \frac{H_{n, 2}}{n 2^{n}} \equiv-\frac{1}{2}\left(B_{p-3}-\frac{B_{p-3}}{4}\right)=-\frac{3}{8} B_{p-3} \quad(\bmod p) .
$$

Now we show (1.1). Observe that

$$
\begin{aligned}
\sum_{k=1}^{p-1} \frac{H_{k}}{k 2^{k}} & =\sum_{1 \leqslant j \leqslant k \leqslant p-1} \frac{1}{j k 2^{k}}=\sum_{1 \leqslant j \leqslant k \leqslant p-1} \frac{1}{(p-k)(p-j) 2^{p-j}} \\
& =\sum_{1 \leqslant j \leqslant k \leqslant p-1} \frac{2^{j-p}(p+j)(p+k)}{\left(p^{2}-j^{2}\right)\left(p^{2}-k^{2}\right)} \\
& \equiv \sum_{1 \leqslant j \leqslant k \leqslant p-1} \frac{2^{j-p}(j k+p(j+k))}{j^{2} k^{2}} \\
& \equiv 2^{-p} \sum_{1 \leqslant j \leqslant k \leqslant p-1} \frac{2^{j}}{j k}+\frac{p}{2} \sum_{1 \leqslant j \leqslant k \leqslant p-1} \frac{2^{j}(j+k)}{j^{2} k^{2}}\left(\bmod p^{2}\right) .
\end{aligned}
$$

In view of Lemmas 2.2 and 2.1,

$$
\begin{aligned}
\sum_{1 \leqslant j \leqslant k \leqslant p-1} \frac{2^{j}-1}{j k} & =\sum_{1 \leqslant j \leqslant k \leqslant p-1} \frac{1}{j k} \sum_{i=1}^{j}\left(\begin{array}{l}
j \\
i
\end{array}\right)=\sum_{i=1}^{p-1} \frac{1}{i} \sum_{k=1}^{p-1} \frac{1}{k} \sum_{j=1}^{k}\left(\begin{array}{c}
j-1 \\
i-1
\end{array}\right) \\
& =\sum_{i=1}^{p-1} \frac{1}{i} \sum_{k=1}^{p-1} \frac{1}{k}\left(\begin{array}{c}
k \\
i
\end{array}\right)=\sum_{i=1}^{p-1} \frac{1}{i^{2}} \sum_{k=1}^{p-1}\left(\begin{array}{c}
k-1 \\
i-1
\end{array}\right) \\
& =\sum_{i=1}^{p-1} \frac{1}{i^{2}}\left(\begin{array}{c}
p-1 \\
i
\end{array}\right)=\sum_{i=1}^{p-1} \frac{(-1)^{i}}{i^{2}} \prod_{r=1}^{i}\left(1-\frac{p}{r}\right) \\
& \equiv \sum_{i=1}^{p-1} \frac{(-1)^{i}\left(1-p H_{i}\right)}{i^{2}} \equiv \frac{p}{2} B_{p-3}-p\left(-\frac{B_{p-3}}{4}\right)\left(\bmod p^{2}\right) .
\end{aligned}
$$


Note that

$$
\sum_{1 \leqslant j \leqslant k \leqslant p-1} \frac{1}{j k}=\sum_{k=1}^{p-1} \frac{H_{k}}{k} \equiv \frac{p}{3} B_{p-3} \quad\left(\bmod p^{2}\right)
$$

by $(2.2)$. Combining the above with $(2.5)$, we finally obtain that

$$
\begin{aligned}
\sum_{k=1}^{p-1} \frac{H_{k}}{k 2^{k}} & \equiv 2^{-p}\left(\frac{3}{4} p B_{p-3}+\frac{p}{3} B_{p-3}\right)+\frac{p}{2} \sum_{k=1}^{p-1} \frac{(-1)^{k}}{k^{3}} \\
& \equiv \frac{13}{24} p B_{p-3}+\frac{p}{2}\left(-\frac{B_{p-3}}{2}\right)=\frac{7}{24} p B_{p-3}\left(\bmod p^{2}\right) \quad(\text { by }(2.1)) .
\end{aligned}
$$

This concludes the proof.

\section{Proof of Theorem 1.2}

Lemma 3.1. Let $p>3$ be a prime and let $m$ be a positive integer with $p-1 \nmid 3 m$. Then

$$
\sum_{1 \leqslant j<k \leqslant p-1}\left(\frac{1}{j^{m} k^{2 m}}+\frac{1}{j^{2 m} k^{m}}\right) \equiv 0 \quad(\bmod p) .
$$

Moreover, if $p>3 m+1$, then

$$
\sum_{1 \leqslant j<k \leqslant p-1}\left(\frac{1}{j^{m} k^{2 m}}+\frac{1}{j^{2 m} k^{m}}\right) \equiv-p \frac{3 m}{3 m+1} B_{p-1-3 m} \quad\left(\bmod p^{2}\right) .
$$

Proof. It is well-known that

$$
\sum_{k=1}^{p-1} \frac{1}{k^{n}} \equiv 0 \quad(\bmod p) \quad \text { for any integer } n \not \equiv 0 \quad(\bmod p-1) .
$$

Also,

$$
\sum_{k=1}^{p-1} \frac{1}{k^{n}} \equiv \frac{p n}{n+1} B_{p-1-n} \quad\left(\bmod p^{2}\right) \quad \text { for } n=1, \ldots, p-2
$$

(see, e.g., [S, Corollary 5.1]). Thus

$\sum_{1 \leqslant j<k \leqslant p-1}\left(\frac{1}{j^{m} k^{2 m}}+\frac{1}{j^{2 m} k^{m}}\right)=\sum_{j=1}^{p-1} \frac{1}{j^{m}} \sum_{k=1}^{p-1} \frac{1}{k^{2 m}}-\sum_{k=1}^{p-1} \frac{1}{k^{3 m}} \equiv 0 \quad(\bmod p)$.

Moreover, we have (3.2) if $p>3 m+1$. 
Lemma 3.2. Let $p>3$ be a prime and let $m$ be a positive even integer. Then

$$
\sum_{1 \leqslant j<k \leqslant p-1}\left(\frac{1}{j^{m} k^{2 m}}-\frac{1}{j^{2 m} k^{m}}\right) \equiv 0 \quad(\bmod p) .
$$

Moreover, if $p>3 m+1$ then

$$
\sum_{1 \leqslant j<k \leqslant p-1}\left(\frac{1}{j^{m} k^{2 m}}-\frac{1}{j^{2 m} k^{m}}\right) \equiv \frac{p m\left(\begin{array}{c}
3 m \\
m
\end{array}\right) B_{p-1-3 m}}{(m+1)(2 m+1)}\left(\bmod p^{2}\right) .
$$

Proof. As $m$ is even, we have

$$
\begin{aligned}
\sum_{1 \leqslant j<k \leqslant p-1} \frac{1}{j^{m} k^{2 m}} & =\sum_{1 \leqslant j<k \leqslant p-1} \frac{1}{(p-k)^{m}(p-j)^{2 m}} \\
& \equiv \sum_{1 \leqslant j<k \leqslant p-1} \frac{1}{j^{2 m} k^{m}}(\bmod p) .
\end{aligned}
$$

Now suppose that $p>3 m+1$. Then

$$
\begin{aligned}
& \sum_{1 \leqslant j<k \leqslant p-1} \frac{1}{j^{m} k^{2 m}}=\sum_{\substack{1 \leqslant j<k \leqslant p-1 \\
j^{2}}} \frac{(p+k)^{m}(p+j)^{2 m}}{\left(p^{2}-k^{2}\right)^{m}\left(p^{2}-j^{2}\right)^{2 m}} \\
\equiv & \sum_{1 \leqslant j<k \leqslant p-1} \frac{\left(k^{m}+p m k^{m-1}\right)\left(j^{2 m}+p 2 m j^{2 m-1}\right)}{j^{4 m} k^{2 m}} \\
\equiv & \sum_{1 \leqslant j<k \leqslant p-1} \frac{1}{j^{2 m} k^{m}}+p m \sum_{1 \leqslant j<k \leqslant p-1}\left(\frac{1}{j^{2 m} k^{m+1}}+\frac{2}{j^{2 m+1} k^{m}}\right)\left(\bmod p^{2}\right) .
\end{aligned}
$$

So, (3.4) is reduced to

$$
\sum_{1 \leqslant j<k \leqslant p-1}\left(\frac{1}{j^{2 m} k^{m+1}}+\frac{2}{j^{2 m+1} k^{m}}\right) \equiv \frac{\left(\begin{array}{c}
3 m \\
m
\end{array}\right) B_{p-1-3 m}}{(m+1)(2 m+1)} \quad(\bmod p) .
$$

Recall that for any integer $n$ we have

$$
\sum_{k=1}^{p-1} k^{n} \equiv \begin{cases}p-1(\bmod p) & \text { if } p-1 \mid n \\ 0(\bmod p) & \text { if } p-1 \nmid n\end{cases}
$$

(See, e.g., [IR, p.235].) Also,

$$
\sum_{j=0}^{k-1} j^{n}=\frac{1}{n+1} \sum_{j=0}^{n}\left(\begin{array}{c}
n+1 \\
j
\end{array}\right) B_{j} k^{n+1-j}
$$


for any $k=1,2,3 \ldots$ and $n=0,1,2, \ldots$ (See, e.g., [IR, p. 230].) Therefore

$$
\begin{aligned}
& \sum_{1 \leqslant j<k \leqslant p-1} \frac{1}{j^{2 m} k^{m+1}} \\
\equiv & \sum_{k=1}^{p-1} \frac{1}{k^{m+1}} \sum_{j=0}^{k-1} j^{p-1-2 m}=\sum_{k=1}^{p-1} \frac{1}{k^{m+1}(p-2 m)} \sum_{j=0}^{p-1-2 m}\left(\begin{array}{c}
p-2 m \\
j
\end{array}\right) B_{j} k^{p-2 m-j} \\
\equiv & -\frac{1}{2 m} \sum_{j=0}^{p-1-2 m}\left(\begin{array}{c}
p-2 m \\
j
\end{array}\right) B_{j} \sum_{k=1}^{p-1} k^{p-1-3 m-j} \\
\equiv & \frac{1}{2 m} \sum_{j=0}^{p-1-2 m}\left(\begin{array}{c}
p-2 m \\
j
\end{array}\right) B_{j}=\frac{1}{2 m}\left(\begin{array}{c}
p-2 m \\
m+1
\end{array}\right) B_{p-1-3 m} \\
\equiv & \frac{1}{2 m}\left(\begin{array}{c}
-2 m \\
m+1 \mid j+3 m
\end{array}\right) B_{p-1-3 m}=\frac{(-1)^{m+1}}{2 m}\left(\begin{array}{c}
3 m \\
m+1
\end{array}\right) B_{p-1-3 m}(\bmod p) .
\end{aligned}
$$

Similarly,

$$
\begin{aligned}
& \sum_{1 \leqslant j<k \leqslant p-1} \frac{1}{j^{2 m+1} k^{m}} \\
\equiv & \sum_{k=1}^{p-1} \frac{1}{k^{m}} \sum_{j=0}^{k-1} j^{p-2-2 m}=\sum_{k=1}^{p-1} \frac{1}{k^{m}(p-1-2 m)} \sum_{j=0}^{p-2-2 m}\left(\begin{array}{c}
p-1-2 m \\
j
\end{array}\right) B_{j} k^{p-1-2 m-j} \\
\equiv & -\frac{1}{2 m+1} \sum_{j=0}^{p-2-2 m}\left(\begin{array}{c}
p-1-2 m \\
j
\end{array}\right) B_{j} \sum_{k=1}^{p-1} k^{p-1-3 m-j} \\
\equiv & \frac{1}{2 m+1} \sum_{j=0}^{p-2-2 m}\left(\begin{array}{c}
p-1-2 m \\
j
\end{array}\right) B_{j}=\frac{1}{2 m+1}\left(\begin{array}{c}
p-1-2 m \\
m
\end{array}\right) B_{p-1-3 m} \\
\equiv & \frac{1}{2 m+1}\left(\begin{array}{c}
-1-2 m \\
m
\end{array}\right) B_{p-1-3 m}=\frac{(-1)^{m}}{2 m+1}\left(\begin{array}{c}
3 m \\
m
\end{array}\right) B_{p-1-3 m}(\bmod p) .
\end{aligned}
$$

Therefore

$$
\begin{aligned}
& \sum_{1 \leqslant j<k \leqslant p-1}\left(\frac{1}{j^{2 m} k^{m+1}}+\frac{2}{j^{2 m+1} k^{m}}\right) \\
\equiv & \left(\frac{(-1)^{m+1}}{2 m}\left(\begin{array}{c}
3 m \\
m+1
\end{array}\right)+2 \frac{(-1)^{m}}{2 m+1}\left(\begin{array}{c}
3 m \\
m
\end{array}\right)\right) B_{p-1-3 m} \\
= & \frac{(-1)^{m}}{(m+1)(2 m+1)}\left(\begin{array}{c}
3 m \\
m
\end{array}\right) B_{p-1-3 m}(\bmod p) .
\end{aligned}
$$


So (3.5) holds as $m$ is even.

Proof of Theorem 1.2. Let $m=2 n$. Clearly

$$
\begin{aligned}
\sum_{k=1}^{p-1} \frac{H_{k, m}^{2}}{k^{m}}= & \sum_{k=1}^{p-1} \frac{1}{k^{m}}\left(\sum_{j=1}^{k} \frac{1}{j^{m}}\right)^{2} \\
= & \sum_{k=1}^{p-1} \frac{1}{k^{m}}\left(\sum_{j=1}^{k} \frac{1}{j^{2 m}}+2 \sum_{1 \leqslant i<j \leqslant k} \frac{1}{i^{m} j^{m}}\right) \\
= & H_{p-1,3 m}+\sum_{1 \leqslant j<k \leqslant p-1} \frac{1}{j^{2 m} k^{m}}+2 \sum_{1 \leqslant i<j \leqslant p-1} \frac{1}{i^{m} j^{2 m}} \\
& +2 \sum_{1 \leqslant i<j<k \leqslant p-1} \frac{1}{i^{m} j^{m} k^{m}}
\end{aligned}
$$

and

$$
\begin{aligned}
H_{p-1, m}^{3} & =\sum_{i=1}^{p-1} \frac{1}{i^{m}}\left(\sum_{k=1}^{p-1} \frac{1}{k^{2 m}}+2 \sum_{1 \leqslant j<k \leqslant p-1} \frac{1}{j^{m} k^{m}}\right) \\
& =H_{p-1,3 m}+3 \sum_{1 \leqslant j<k \leqslant p-1}\left(\frac{1}{j^{2 m} k^{m}}+\frac{1}{j^{m} k^{2 m}}\right)+6 \sum_{1 \leqslant i<j<k \leqslant p-1} \frac{1}{i^{m} j^{m} k^{m}} .
\end{aligned}
$$

As $H_{p-1, m} \equiv 0(\bmod p)$, from the above we obtain

$$
\begin{aligned}
\sum_{k=1}^{p-1} \frac{H_{k, m}^{2}}{k^{m}} \equiv & H_{p-1,3 m}+\sum_{1 \leqslant j<k \leqslant p-1}\left(\frac{1}{j^{2 m} k^{m}}+\frac{2}{j^{m} k^{2 m}}\right) \\
& -\frac{H_{p-1,3 m}}{3}-\sum_{1 \leqslant j<k \leqslant p-1}\left(\frac{1}{j^{2 m} k^{m}}+\frac{1}{j^{m} k^{2 m}}\right) \\
= & \frac{2}{3} H_{p-1,3 m}+\sum_{1 \leqslant j<k \leqslant p-1} \frac{1}{j^{m} k^{2 m}}\left(\bmod p^{2}\right) .
\end{aligned}
$$

Thus, by $(3.1),(3.3)$ and the congruence $H_{p-1,3 m} \equiv 0(\bmod p)$, we immediately get (1.3).

Below we assume that $p>3 m+1$. Adding (3.2) and (3.4) we obtain

$$
\begin{aligned}
2 \sum_{1 \leqslant j<k \leqslant p-1} \frac{1}{j^{m} k^{2 m}} & \equiv p m B_{p-1-3 m}\left(-\frac{3}{3 m+1}+\frac{\left(\begin{array}{c}
3 m \\
m
\end{array}\right)}{(m+1)(2 m+1)}\right) \\
& =\frac{p m}{3 m+1}\left(\frac{\left(\begin{array}{c}
3 m+1 \\
m
\end{array}\right)}{m+1}-3\right) B_{p-1-3 m}\left(\bmod p^{2}\right) .
\end{aligned}
$$


Note also that

$$
H_{p-1-3 m} \equiv p \frac{3 m}{3 m+1} B_{p-1-3 m} \quad\left(\bmod p^{2}\right)
$$

Therefore

$$
\begin{aligned}
\sum_{k=1}^{p-1} \frac{H_{k, m}^{2}}{k^{m}} & \equiv \frac{2}{3} \cdot p \frac{3 m}{3 m+1} B_{p-1-3 m}+\left(\frac{\left(\begin{array}{c}
3 m+1 \\
m
\end{array}\right)}{m+1}-3\right) \frac{p m / 2}{3 m+1} B_{p-1-3 m} \\
& =\left(\frac{\left(\begin{array}{c}
3 m+1 \\
m
\end{array}\right)}{m+1}+1\right) \frac{p m / 2}{3 m+1} B_{p-1-3 m} \\
& =\left(\left(\begin{array}{c}
3 m+1 \\
m-1
\end{array}\right)+\frac{m}{2}\right) \frac{p B_{p-1-3 m}}{3 m+1}\left(\bmod p^{2}\right) .
\end{aligned}
$$

This proves (1.4).

So far we have completed the proof of Theorem 1.2.

Acknowledgment. The authors wish to thank the referee for helpful comments.

\section{REFERENCES}

[C] S. W. Coffman, Problem 1240 and Solution: An infinite series with harmonic numbers, Math. Mag. 60 (1987), 118-119.

[G] H. W. Gould, Combinatorial Identities, Morgantown Printing and Binding Co., 1972.

[H] V. Hernández, Solution IV of problem 10490, Amer. Math. Monthly 106 (1999), 589-590.

[IR] K. Ireland and M. Rosen, A Classical Introduction to Modern Number Theory (Graduate texts in math.; 84), 2nd ed., Springer, New York, 1990.

[M] R. Meštrović, Proof of a congruence for harmonic numbers conjectured by $Z$.- $W$. Sun, Int. J. Number Theory 8 (2012), 1081-1085.

[SW] J. Sondow and E. W. Weisstein, Harmonic Number, MathWorld-A Wolfram Web Resource, http://mathworld.wolfram.com/HarmonicNumber.html.

[S] Z. H. Sun, Congruences concerning Bernoulli numbers and Bernoulli polynomials, Discrete Appl. Math. 105 (2000), 193-223.

[Su] Z. W. Sun, Arithmetic theory of harmonic numbers, Proc. Amer. Math. Soc. 140 (2012), 415-428.

[ST] Z. W. Sun and R. Tauraso, New congruences for central binomial coefficients, Adv. in Appl. Math. 45 (2010), 125-148.

[W] J. Wolstenholme, On certain properties of prime numbers, Quart. J. Math. 5 (1862), 35-39.

[ZS] L. L. Zhao and Z. W. Sun, Some curious congruences modulo primes, J. Number Theory 130 (2010), 930-935. 